\title{
Effect of Low-intensity Exercise on Physical and Cognitive Health in Older Adults: a Systematic Review
}

\author{
Andy C. Y. Tse ${ }^{1 *}$, Thomson W. L. Wong ${ }^{2}$ and Paul H. Lee ${ }^{3}$
}

\begin{abstract}
Background: It is well known that physical exercise is important to promote physical and cognitive health in older population. However, inconsistent research findings were shown regarding exercise intensity, particularly on whether low-intensity exercise (1.5 metabolic equivalent tasks (METs) to 3.0 METs) can improve physical and cognitive health of older adults. This systematic review aimed to fill this research gap. The objective of this study is to conduct a systematic review of the effectiveness of low-intensity exercise interventions on physical and cognitive health of older adults.

Methods: Published research was identified in various databases including CINAHL, MEDLINE, PEDro, PubMed, Science Direct, SPORTDiscus, and Web of Science. Research studies published from January 01, 1994 to February 01, 2015 were selected for examination. Studies were included if they were published in an academic peer-reviewed journal, published in English, conducted as randomized controlled trial (RCT) or quasi-experimental studies with appropriate comparison groups, targeted participants aged 65 or above, and prescribed with low-intensity exercise in at least one study arm. Two reviewers independently extracted the data (study, design, participants, intervention, and results) and assessed the quality of the selected studies. Fifteen studies met the inclusion criteria. Quality index ranged from 15 to 18 mean $=18.3$ with a full score of 28 , indicating a moderate quality. Most of the outcomes reported in these studied were lower limb muscle strength $(n=9)$, balancing $(n=7)$, flexibility $(n=4)$, and depressive symptoms $(n=3)$.
\end{abstract}

Results: Out of the 15 selected studies, 11 reported improvement in flexibility, balancing, lower limb muscle strength, or depressive symptoms by low-intensity exercises.

Conclusions: The current literature suggests the effectiveness of low-intensity exercise on improved physical and cognitive health for older adults. It may be a desired intensity level in promoting health among older adults with better compliance, lower risk of injuries, and long-term sustainability.

Keywords: Low-intensity exercise; Physical health; Cognitive health; Older adults

\section{Key Points}

- Low-intensity exercise offers both physical and cognitive health benefits to older adults.

- Low-intensity exercise is useful to induce health benefits for high-risk population such as physical frail older adults.

\footnotetext{
* Correspondence: andytcy@ied.edu.hk

'Department of Health and Physical Education, Hong Kong Institute of Education, Tai Po, Hong Kong, China

Full list of author information is available at the end of the article
}

- Low-intensity exercise induces better exercise adherence as relative to moderate and high intensity exercise.

\section{Background}

In recent decades, many parts of the world have aging populations, including the UK, Canada, and the USA [1-7]. Hong Kong is no exception. It is estimated that the number of adults aged 65 and older in Hong Kong will increase by 1.6 million to 3.6 million by 2041 -with approximately one in three persons being older adults,

\section{它 Springer}


up from the current proportion of one in seven [8]. With advancing age and declining functional capacity [9], older adults are more prone to health-related problems such as declining muscular strength and cardiovascular endurance $[10,11]$. A survey conducted in Hong Kong revealed that $70.4 \%$ of older adults reported to suffer from at least one chronic disease [12], which describes a high rate of morbidity and mortality among older adults [13, 14]. Moreover, with declining cognitive functions, risk of dementia and severity of depressive symptoms were unsurprisingly increased [15-21].

With such a dramatic increase in the older adult population, one may foresee that medical costs associated with older adults will inevitably continue to grow. According to the Hong Kong Government, people aged 65 years and over constituted $13.2 \%$ of the whole Hong Kong population but consumed $15.8 \%$ of total government expenditure in the 2013-2014 financial year [22]. This situation presents challenges to various healthcare service providers for older adults. The search for optimal preventive care and public health interventions that promote physical and cognitive health among aging populations is thus crucial for city planners, healthcare professionals, and stakeholders.

Exercise is one such preventive public health intervention. It is widely reported to be effective in reducing all-cause mortality, cardiovascular disease, and cancer [23-28]. The term "exercise" is defined as a regular structured program of physical activity [29,30], where "physical activity" is defined as an activity in daily life that may be categorized as occupational, sports, conditioning, household, or other [29]. Previous studies have shown that exercise can change postural control functioning in older adults, which leads to a reduced fall risk and better maintenance of upright stances [31-34]. In addition, exercise is associated with cognitive health in older adults by delaying the symptoms of cognitive diseases, such as dementia, and mood disorders, such as depression [35-37].

Although the benefits of exercise have been well documented in the literature, there is a lack of universal agreement on the frequency, intensity, and types of exercise required for health promotion among older adults. "Exercise intensity" is defined as how hard the exertion is during exercise [38] and is typically measured in metabolic equivalent task (MET) [39]. One MET is defined as the rate of energy expenditure at rest [40]. Activities with METs between 3.0 and 6.0 are considered to have moderate intensity, whereas exercise intensities above 1.5 METs and below 3.0 METs are considered to be low [38, 40, 41]. Typical low-intensity exercises for older adults include light walking, stretching, lifting hand weights, sit-ups, and push-ups against the wall [42]. Combination exercises with low intensity are often administered as exercise programs for older adults [43]. Currently, considerable research has shown that activities with at least moderate intensity (including running, tennis, and aerobics) could lower the risk of all-cause mortality, type 2 diabetes, hypertension, stroke, colon cancer, breast cancer, depressive symptoms, and dementia [44-48]. In contrast, the effects of low-intensity exercise-both lower in injury risk and generally more affordable to older adults-did not receive the same attention as did those of moderate intensity exercises. Previous literature on the effectiveness of low-intensity exercise in older adults has shown conflicting evidence. One study showed that low-intensity exercise was not associated with health improvements [49]; however, others have demonstrated significant improvement in health [50, 51]. Consequently, it remains debatable whether low-intensity exercise would be effective in improving physical and cognitive health in older adults. The purpose of the present study was to conduct a systematic review to draw a conclusion about the effectiveness of low-intensity exercise in older adults.

\section{Methods}

\section{Information Sources}

This review adhered to the Preferred Reporting Items for Systematic Reviews and Meta-Analysis (PRISMA) guidelines [52]. Six electronic databases (CINAHL, MEDLINE/ PubMed, PEDro, Science Direct, SPORTDiscus, and Web of Science) were used to access as many relevant articles as possible.

\section{Search Strategy and Data Items}

A systematic search strategy was conducted using the electronic databases with varying combinations of the following terms found in the title, abstract, or keyword fields: "exercise OR low intensity exercise," "health OR physical exercise OR cognitive exercise," and "older adult OR elderly." For example, "exercise OR "low intensity exercise' AND health AND 'older adult" was searched in the CINAHL electronic database, and 218 relevant studies were found. Searches included papers published from 1994 to March 01, 2015.

\section{Eligibility Criteria and Study Selection}

To be included in this systematic review, articles were required to be as follows: published in an academic, peer-reviewed journal; published in English; conducted as a randomized controlled trial (RCT) or as a quasiexperimental study with appropriate comparison groups; targeted to participants aged 65 or older; and inclusive of an exercise intervention of differing intensity levels, with low-intensity exercise in at least 1 study arm. The low-intensity exercise referred to any exercise level greater than 1.5 METs but less than 3 METs [38, 40]. 
There was no limit imposed on the duration, frequency, or type of exercise intervention, and no specific physical or cognitive outcome measures were stipulated.

\section{Data Extraction and Quality Assessment}

The included studies were analyzed and cross-checked independently by two authors to extract the following information: study design, objectives and discussion, participants and eligibility criteria, intervention used, variables and measurement, key outcomes, and conclusions. A data extraction form was used to standardize the data extraction process. Discussion was conducted in cases of disagreement, and a consensus would be reached on whether the studies in question would be included in the present review.

The two reviewers independently assessed the quality of the articles with the use of the quality index (QI) [53] shown in Appendix 1. The quality index is a wellestablished quality assessment tool for systematic reviews and social care interventions. It comprises 27 items that are categorized into five subscales: reporting (10 items), external validity (3 items), internal validity-bias (7 items), internal validity-confounding (6 items), and power (1 item) [53]. All item answers are indicated as "yes" (score $=1)$, "no" (score $=0)$, or "unable to determine" (score $=\mathrm{U})$. A higher score represents higher quality. In cases of disagreement in this review, a third reviewer was consulted to resolve any discrepancies.

\section{Data Synthesis}

The selected studies were divided into two domains-physical health and cognitive health. Within each domain, the low-intensity exercise group was compared either with a control or another exercise group.

\section{Results}

\section{Search Results}

A total of 2884 relevant published studies were initially found from searches, as shown in Fig. 1. After initial screening of abstracts against the inclusion criteria, 337 studies remained. After final analysis, 15 of those studies [54-68] met the inclusion criteria, with articles from CINAHL $(n=2)$, MEDLINE/PubMed $(n=2$; included studies from MEDLINE and PubMed were identical), PEDro $(n=7)$, Science Direct $(n=2)$, SPORTDiscus $(n=1)$, and Web of Science $(n=1)$.

\section{Quality Assessment}

Quality index scores ranged from 15 to $20($ mean $=18.3)$, with the highest possible score being 28. Most studies $(n=12)$ scored 18 or higher [54-56, 59-63, 65-68], whereas the rest $(n=3)$ had QI scores of 15 [64], 16 [57], and 17 [58] (Table 1). Inter-rater reliability of both raters was assessed by comparing the total rated scores with the means of the Spearman correlation coefficients and the level of agreement with the $\kappa$ statistic. Interrater reliability was moderate $(Y=0.61 ; \kappa=0.57)$ [53]. As shown in Table 1, most studies were very clear on reporting and satisfied the criteria of external validity. The most diversifying issues were related to internal validity-confounding criteria such as trials not being blinded or open-labeled, inadequate adjustment for confounding in estimating the effect of low-intensity exercise, and lack of reporting on participant attrition.

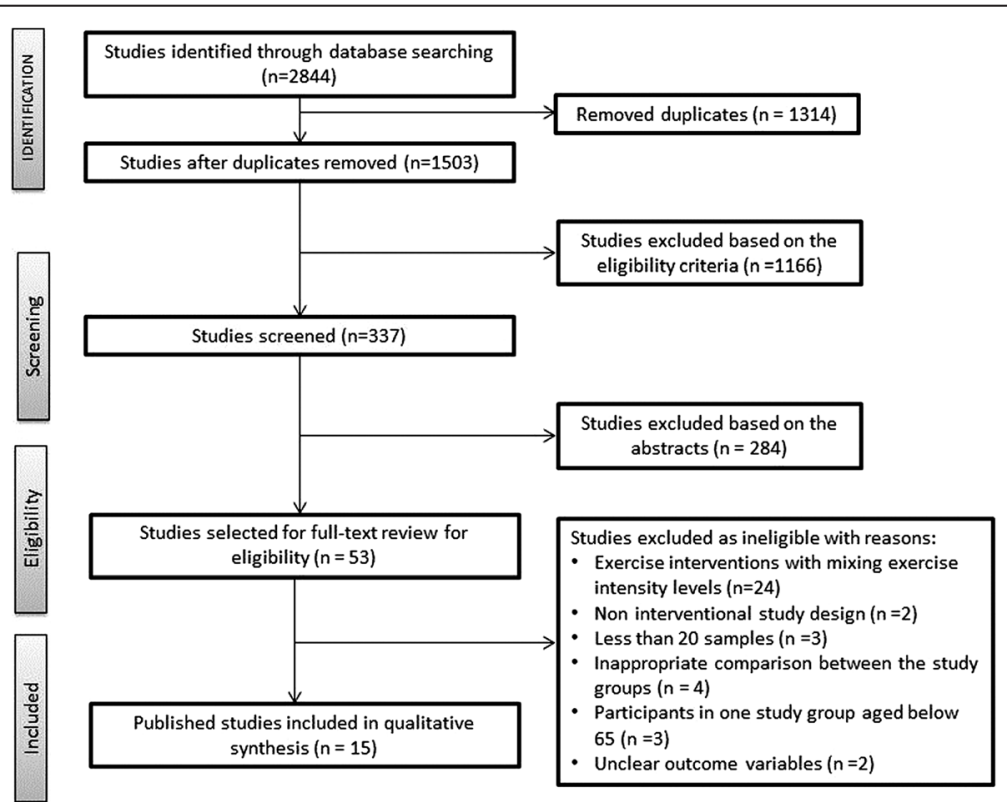

Fig. 1 Flowchart describing the selection process of the included studies 
Table 1 Quality index assessment scale ratings

\begin{tabular}{|c|c|c|c|c|c|c|}
\hline \multirow[t]{2}{*}{ Study } & Reporting & External validity & Internal validity_bias & Internal validity_confounding & Power & Ql score \\
\hline & Full score, 11 & Full score, 3 & Full score, 7 & Full score, 6 & Full score, 1 & Full score, 28 \\
\hline Blair et al., 2014 [54] & 7 & 2 & 5 & 4 & 0 & 18 \\
\hline Brown et al., 2000 [55] & 8 & 3 & 5 & 3 & 0 & 19 \\
\hline Dawe et al., 1995 [56] & 7 & 3 & 5 & 4 & 0 & 19 \\
\hline DeVito et al., 2003 [57] & 6 & 3 & 5 & 2 & 0 & 16 \\
\hline Lam et al., 2011 [58] & 8 & 2 & 4 & 3 & 0 & 17 \\
\hline Li et al., 2005 [59] & 8 & 3 & 5 & 2 & 0 & 18 \\
\hline Mangione et al., 1999 [60] & 8 & 3 & 5 & 3 & 0 & 19 \\
\hline Means et al., 1996 [61] & 9 & 3 & 4 & 2 & 0 & 18 \\
\hline Morey et al., 2009 [62] & 7 & 2 & 6 & 3 & 0 & 18 \\
\hline Morgan et al., 2004 [63] & 8 & 3 & 5 & 3 & 0 & 19 \\
\hline Motl et al., 2005 [64] & 6 & 3 & 4 & 2 & 0 & 15 \\
\hline Rosie et al., 2007 [65] & 9 & 3 & 5 & 2 & 0 & 19 \\
\hline Schnelle et al., 2003 [66] & 8 & 3 & 6 & 3 & 0 & 20 \\
\hline Singh et al., 2005 [67] & 8 & 3 & 6 & 3 & 0 & 20 \\
\hline Wolfson et al., 1996 [68] & 9 & 3 & 5 & 3 & 0 & 20 \\
\hline
\end{tabular}

\section{Study Characteristics}

All included studies $(n=15)$ were pre- and posttest designs, 10 of which $[55,58,59,62,63,65-68]$ were RCTs, and 4 of which $[54,59,64,68]$ included follow-up assessments after the intervention had been completed (Table 2). Sample sizes varied from 20 [55] to 641 [61]. Most of the studies $(n=12)$ were related to the physical health of older adults, and 4 were related to cognitive health (Table 2). All studies used low-intensity exercise of differing types as the study intervention, including lowintensity stationary cycling [59], stretching [56, 59, 61, 64], walking $[56,61,63]$, Tai Chi $[58,59,68]$, balance training $[63,68]$, resistance training $[64,67,68]$, seated exercise $[56,63]$, and functional exercise programs $[55,57,62,66]$. Control groups engaging in their usual physical activities were used in 6 studies [57, 58, 63, 66-68], and 1 study used a viewing of a 15-min exercise program video [58]. In the remaining studies, the intervention groups were compared with other exercise groups such as stretching and toning groups [61], non-obstacle practice groups [63], high-intensity resistance training groups [69], home-based exercise groups [57], delayed intervention groups [56, 64], high-intensity cycling groups [62], and knee extension groups [67]. The duration and frequency of the lowintensity exercise intervention were diverse, ranging from $1 \mathrm{~h}$ to 1 year and from one to three times per week.

\section{Physical and Cognitive Health Outcomes}

In terms of physical health, improvements were reported in range of motion [57], endurance [61, 62], gait velocity $[60,68]$, lower limb muscle strength [53-56, 61, 62, 66, 68], overall pain [60], balance [55. 57, 61, 66-68], and peak oxygen consumption $[56,66]$. In terms of cognitive health, significant reduction in depression scores $[64,67]$ and improved cognitive functions $[56,58]$ were reported. Overall, the key outcomes of the included studies were lower limb muscle strength, flexibility, balance, and depressive symptoms.

\section{Discussion}

This systematic review examined the effect of lowintensity exercise on physical and cognitive health in older adults. The majority of the studies included showed that low-intensity exercise was effective in improving balance and lower limb muscle strength, as was evident in the reduction of fall frequency and fall risk $[59,61,63,66,68]$.

Falls are a major cause of morbidity and mortality in older adult populations [69-71]. A previous study showed that the fall rate among community-dwelling older adults aged 65 or older was $26.4 \%$, and the incidence rate of new fallers was 198 per 1000 persons per year [72]. Serious injuries such as bone fractures [72-74] commonly result from falls. Even for those who did not experience any serious injuries after a fall, the resultant functional deterioration [75, 76], self-rated health [77], and fear of falling [78, 79] may lead to impairment of daily living activities, adversely affecting quality of life.

More than half of the included studies $(n=10)$ supported the benefits of low-intensity exercise intervention towards fall prevention in older adults. Brown et al. [55] designed a 3-month program of low-intensity exercises for older adult participants with minor frailty (Table 2). Participants completed a modified physical performance 
Table 2 Description of studies included in the review

\begin{tabular}{|c|c|c|c|c|c|}
\hline Study & Domain & Design & Sample & Intervention & Key outcomes \\
\hline \multirow[t]{8}{*}{ Dawe et al., 1995 [56] } & \multirow[t]{8}{*}{ Cognitive health } & \multirow[t]{8}{*}{ Pre-post test } & $N=20$ & Duration, $1 \mathrm{~h}$ & Blood pressure $=\mathrm{mmHg}$ \\
\hline & & & $\begin{array}{l}\text { Nursing home } \\
\text { residents }\end{array}$ & \multirow{3}{*}{$\begin{array}{l}\text { Intervention group = received } \\
\text { the Canadian Red Cross } \\
\text { Society's Senior's Fun and } \\
\text { Fitness program. }\end{array}$} & Pulse $=$ beats $/ \mathrm{min}$ \\
\hline & & & $\begin{array}{l}\text { Cognitively } \\
\text { unimpaired }\end{array}$ & & Three cognitive tests: \\
\hline & & & Male, 4; Female, 16 & & 1. Set test $=$ number of words \\
\hline & & & $\begin{array}{l}\text { Intervention group } \\
(n=10)\end{array}$ & \multirow{3}{*}{$\begin{array}{l}\text { Control group }=\text { viewed a } \\
15 \text {-min video of low-intensity } \\
\text { exercise program }\end{array}$} & \\
\hline & & & Mean age: 83.9 & & $\begin{array}{l}\text { 2. Word fluency test }=\text { number } \\
\text { of words correctly recalled; }\end{array}$ \\
\hline & & & $\begin{array}{l}\text { Control group } \\
(n=10)\end{array}$ & & \\
\hline & & & Mean age $=85.1$ & $\begin{array}{l}\text { Overall intervention } \\
\text { compliance }=\text { no information }\end{array}$ & $\begin{array}{l}\text { 3. Symbol digit test = number } \\
\text { of corrected digit encoding }\end{array}$ \\
\hline \multirow[t]{10}{*}{ Means et al., 1996 [61] } & \multirow[t]{10}{*}{ Physical health } & \multirow[t]{10}{*}{ Pre-post test } & $N=65$ & Duration, 6 weeks & \multirow{3}{*}{$\begin{array}{l}\text { Performance score }(0=\text { poorest } \\
\text { performance to } 3=\text { best } \\
\text { performance; total point }=36) \\
\text { and completion time (in seconds) } \\
\text { on a functionally oriented obstacle } \\
\text { course }\end{array}$} \\
\hline & & & Community-dwelling; & $\begin{array}{l}\text { Balance and mobility exercise } \\
\text { protocol: }\end{array}$ & \\
\hline & & & $\begin{array}{l}\text { One or more falls } \\
\text { within a year prior to } \\
\text { the study }\end{array}$ & \multirow{2}{*}{$\begin{array}{l}\text { Active stretching, postural } \\
\text { control, endurance walking, } \\
\text { repetitive muscle coordination } \\
\text { exercises }\end{array}$} & \\
\hline & & & Gender: no specified & & $\begin{array}{l}\text { Self-reported falls = number } \\
\text { of falls }\end{array}$ \\
\hline & & & & Cognitive intervention sessions & Fall related injuries \\
\hline & & & $\begin{array}{l}\text { Intervention group } \\
(n=31)\end{array}$ & \multirow{3}{*}{$\begin{array}{l}\text { Practice group = received extra } \\
\text { training on an obstacle course } \\
\text { along with the exercise } \\
\text { protocol }\end{array}$} & \\
\hline & & & Mean age: 75 & & \\
\hline & & & $\begin{array}{l}\text { Control group } \\
(n=34)\end{array}$ & & \\
\hline & & & \multirow[t]{2}{*}{ Mean age, 75} & $\begin{array}{l}\text { Non-practice group = receive } \\
\text { no training on an obstacle } \\
\text { course but only the exercise } \\
\text { protocol }\end{array}$ & \\
\hline & & & & $\begin{array}{l}\text { Overall intervention } \\
\text { compliance = no information }\end{array}$ & \\
\hline \multirow[t]{2}{*}{ Wolfson et al., 1996 [68] } & \multirow[t]{2}{*}{ Physical health } & \multirow[t]{2}{*}{$\mathrm{RCT}$} & $N=110$ & Phase 1 & \multirow{2}{*}{$\begin{array}{l}\text { Loss of balance during sensory } \\
\text { organization test }(L O B)=\text { number } \\
\text { of times that participants received } \\
\text { support from the experimenter }\end{array}$} \\
\hline & & & $\begin{array}{l}\text { Community-dwelling } \\
\text { Healthy }\end{array}$ & $\begin{array}{l}\text { 3-month balance and strength } \\
\text { training were provided to the }\end{array}$ & \\
\hline
\end{tabular}

Intervention group = significantly

Intervention group s significantly

increased pulse rate (from 69.2 to

71.2 beats $/ \mathrm{min}$ ) and blood pressure

(from $140 / 75$ to $145 / 73 \mathrm{mmHg}$ )

$\left(p^{\prime} s<0.001\right)$

Control group $=$ no differences in pulse rate (between 74.1 to 74.7

beats/min), blood pressure (from

$137 / 74$ to $136 / 72 \mathrm{mmHg}$ ), and three

cognitive tests

(p's > 0.05)

Between groups, intervention group

= showed a better cognitive

performance (i.e., improved recall

ability in the Set test (40 words

to 46 words) than the control group

to 46 words) than the control group
( 43 words to 44 words) $(p<0.05)$ )

Practice group $=$ significantly

decreased the completion time (440.9 to 351.6 s); increased in performance score (24.6 to 26.4) $(p ' s<0.05)$

Non-practice group = significantly decreased the completion time (319.1 to 293.5 secs); increased in performance score (25.7 to 26.7) $(p ' s<0.05)$

Between groups = no significant differences in the completion time performance score $(p>0.05)$

Between groups $=$ no significant difference in number of falls $(p>0.05)$

Overall, all participants = decreased the completion time (378 to 321

secs); improved in mean

performance scores (from 25.2

to 26.5 points) after the exercise protocols

Balance group $=$ significant mprovements in LOB (3.6 to 1.4), FBOS (0.44 to $0.52 \%$ of foot length), Healthy support from the experimenter 
Table 2 Description of studies included in the review (Continued)

\begin{tabular}{|c|c|c|c|c|c|c|}
\hline & & & Male, 64; Female, 46 & $\begin{array}{l}\text { respective groups (45-min per } \\
\text { week) }\end{array}$ & & $\begin{array}{l}\text { no improvement in ISOK ( } 8.0 \text { to } \\
8.1 \mathrm{Nm} / \mathrm{kg}) \text { and UGV }(1.14 \text { to } \\
1.18 \mathrm{~m} / \mathrm{s})\left(p^{\prime} s>0.05\right)\end{array}$ \\
\hline & & & $\begin{array}{l}\text { Balance group } \\
(n=28)\end{array}$ & \multirow{2}{*}{$\begin{array}{l}\text { Control group was } \\
\text { encouraged to continue their } \\
\text { usual activities }\end{array}$} & \multirow[t]{2}{*}{$\begin{array}{l}\text { Functional Base of Support } \\
\text { (FBOS) }=\% \text { of foot length }\end{array}$} & \multirow{4}{*}{$\begin{array}{l}\text { Strength group = significant } \\
\text { improvements in ISOK ( } 6.5 \text { to } \\
8.0 \mathrm{Nm} / \mathrm{kg} \text { ) and LOB ( } 3.7 \text { to } 2.1) \\
\left.\text { ( } p^{\prime}<0.001\right) \text {; no improvements in } \\
\text { other measures: FBOS (0.38 to } 0.39 \% \\
\text { of foot length), and SST }(9.1 \text { to } 10.0 \\
\text { secs), and UGV (1.08 to } 1.17 \mathrm{~m} / \mathrm{s}) \\
\left(p^{\prime} s>0.05\right)\end{array}$} \\
\hline & & & Mean age, 78.9 & & & \\
\hline & & & Strength group & \multirow[t]{2}{*}{ Phase 2} & Single Stance Time $(S S T)=$ seconds & \\
\hline & & & $(n=28)$ & & Isokinetic strength (ISOK) = Nm/kg & \\
\hline & & & Mean age, 80.0 & \multirow{4}{*}{$\begin{array}{l}\text { 6-month low-intensity balance } \\
\text { and strength maintenance } \\
\text { programe (Tai Chi training } \\
\text { with self-administered home } \\
\text { practice) ( } 1 \text { hour per week) }\end{array}$} & \multirow[t]{5}{*}{ Usual Gait Velocity $(U G V)=\mathrm{m} / \mathrm{s}$} & \multirow{4}{*}{$\begin{array}{l}\text { Balance and strength group }= \\
\text { significant improvements in LOB } \\
\text { ( } 3.6 \text { to } 1.9), \text { FBOS }(0.4 \text { to } 0.5 \% \text { of } \\
\text { foot length), SST ( } 5.4 \text { to } 15.1 \text { secs), } \\
\text { ISOK ( } 6.8 \text { to } 8.0 \mathrm{Nm} / \mathrm{kg})\left(p^{\prime} s<0.001\right) \text {; } \\
\text { no significant improvement in UGV } \\
\text { (1.12 to } 1.09 \mathrm{~m} / \mathrm{s})\left(p^{\prime} s>0.05\right)\end{array}$} \\
\hline & & & $\begin{array}{l}\text { Balance and strength } \\
\text { group }(n=27)\end{array}$ & & & \\
\hline & & & Mean age, 79.7 & & & \\
\hline & & & $\begin{array}{l}\text { Control group } \\
(n=27)\end{array}$ & & & \\
\hline & & & Mean age, 80.6 & $\begin{array}{l}\text { Overall intervention } \\
\text { compliance }=72 \%\end{array}$ & & $\begin{array}{l}\text { Overall, no group differences } \\
\left(p^{\prime} s>0.10\right)\end{array}$ \\
\hline \multirow[t]{13}{*}{ Mangione et al., 1999 [60] } & \multirow[t]{13}{*}{ Physical health } & \multirow[t]{13}{*}{ Pre-post test } & \multirow[t]{7}{*}{$N=39$} & Duration, 10 weeks & Timed chair rise $=$ second & \multirow{4}{*}{$\begin{array}{l}\text { High intensity group = significantly } \\
\text { reduced the chair rise time ( } 23.5 \text { to } \\
19.3 \text { secs) and AlMS2 pain score ( } 4.3 \\
\text { to } 3.0) \text {; significantly increased in } 6- \\
\text { min walk ( } 488.0 \text { to } 540.6 \text { m), aerobic } \\
\text { capacity (11.0 to } 13.3 \text { min), and peak } \\
\text { oxygen }\end{array}$} \\
\hline & & & & \multirow{2}{*}{$\begin{array}{l}\text { Exercise training = Stationary } \\
\text { cycling; } 1 \text { hour each session; } \\
\text { cycle } 25 \text { min; } 3 \text { times per week }\end{array}$} & 6 -min walk test $=\mathrm{m}$ & \\
\hline & & & & & \multirow{2}{*}{$\begin{array}{l}\text { Arthritis Impact Measurement Scale } 2 \\
\text { (AlMS2) pain score }\end{array}$} & \\
\hline & & & & \multirow{2}{*}{$\begin{array}{l}\text { High intensity group }= \\
\text { stationary cycling at } 70 \% \\
\text { heart rate reserve }\end{array}$} & & \\
\hline & & & & & \multirow{2}{*}{$\begin{array}{l}\text { Aerobic capacity time for graded } \\
\text { exercise test }=\text { min }\end{array}$} & \multirow{2}{*}{$\begin{array}{l}\text { consumption }(1454.1 \text { to } \\
1545.3 \mathrm{ml} / \mathrm{min})\left(p^{\prime} s<0.01\right)\end{array}$} \\
\hline & & & & \multirow{2}{*}{$\begin{array}{l}\text { Low-intensity group }= \\
\text { stationary cycling at } 40 \% \\
\text { heart rate reserve }\end{array}$} & & \\
\hline & & & & & \multirow[t]{7}{*}{ Peak oxygen consumption = ml/min } & \multirow{7}{*}{$\begin{array}{l}\text { Low-intensity group }=\text { significantly } \\
\text { reduced the chair rise time ( } 23.1 \text { to } \\
19.0 \text { secs) and AlMS2 pain score } \\
\text { (3.6 to 3.1); significantly increased in } \\
6 \text {-min walk ( } 491.1 \text { to } 526.9 \mathrm{~m}) \text {, } \\
\text { aerobic capacity time }(11.1 \text { to } \\
13.0 \mathrm{~min}) \text {, and peak oxygen } \\
\text { consumption }(1710.2 \text { to } \\
1807.3 \mathrm{ml} / \mathrm{min})\left(p^{\prime} s<0.01\right)\end{array}$} \\
\hline & & & $\begin{array}{l}\text { Suffered from knee } \\
\text { osteoarthritis }\end{array}$ & \multirow[t]{6}{*}{$\begin{array}{l}\text { Overall intervention } \\
\text { compliance = no information }\end{array}$} & & \\
\hline & & & Community-dwelling & & & \\
\hline & & & Male, 13; Female, 26 & & & \\
\hline & & & $\begin{array}{l}\text { High intensity cycling } \\
\text { group }(n=19) \text { mean } \\
\text { age }=71.1\end{array}$ & & & \\
\hline & & & $\begin{array}{l}\text { Low-intensity cycling } \\
\text { group }(n=20)\end{array}$ & & & \\
\hline & & & Mean age $=71.0$ & & & \\
\hline Study & Domain & Design & Sample & Intervention & Key outcomes & Results \\
\hline Brown et al., 2000 [55] & Physical health & $\mathrm{RCT}$ & $N=87$ & Duration, 3 months & $\begin{array}{l}\text { Physical Performance Test (PPT) } \\
\text { score }\end{array}$ & $\begin{array}{l}\text { EXER group = significant } \\
\text { improvements on the PPT score }\end{array}$ \\
\hline
\end{tabular}


Table 2 Description of studies included in the review (Continued)

\begin{tabular}{|c|c|c|c|c|c|c|}
\hline & & & Community-dwelling & Supervised exercise group $=$ & Strength measures $=\mathrm{ft} / \mathrm{lb}$ & (29 to 31 points), strength measures \\
\hline & & & $<32$ points on & $\begin{array}{l}\text { low-intensity supervised exer- } \\
\text { cise program ( } 22 \text { exercises; } 3\end{array}$ & Range of motion $=\mathrm{mm}$ & $\begin{array}{l}\text { (e.g., isometric knee extension: } 62 \text { to } \\
65 \mathrm{ft} / \mathrm{lb} \text { ), ranged of motion (e.q. }\end{array}$ \\
\hline & & & $\begin{array}{l}\text { Physical Performance } \\
\text { Test (PPT) }\end{array}$ & $\begin{array}{l}\text { times per week) targeting all } \\
\text { muscle groups }\end{array}$ & Balance measures: & $\begin{array}{l}\text { shoulder flexion: } 160 \text { to } 165 \mathrm{~mm} \text { ), } \\
\text { balance measures (e.g. one-limb }\end{array}$ \\
\hline & & & Male, 20; Female, 28 & & Obstacle course = second; functional & stand: 4.1 to $7.6 \mathrm{~s}$ ), and coordination \\
\hline & & & $\begin{array}{l}\text { Supervised exercise } \\
\text { group (EXER) }(n=48)\end{array}$ & $\begin{array}{l}\text { Home-based flexibility activity } \\
\text { group = some of the exercises } \\
\text { done in the other group and }\end{array}$ & $\begin{array}{l}\text { reach = inch; Romberg = second; } \\
\text { one-limb stand = second; balance } \\
\text { beam = second) }\end{array}$ & $\begin{array}{l}<0.05) \text {; no significant improvements } \\
\text { in gait measures }(p>0.05)\end{array}$ \\
\hline & & & & were not supervised. & Gait measures : gait velocity = & HOME group $=$ no significant \\
\hline & & & Mean age, 83 & Overall intervention & $\begin{array}{l}\mathrm{m} / \mathrm{min} \text {; stride length }=\mathrm{m} \text {; stance } \\
\text { time = second; swing }=\% \text { of gait }\end{array}$ & $\begin{array}{l}\text { Improvements on } 191 \text { score ( } 29 \text { to } \\
29 \text { points), strength measures (e.g., }\end{array}$ \\
\hline & & & $\begin{array}{l}\text { Home-based } \\
\text { flexibility activity }\end{array}$ & ormation & $\begin{array}{l}\text { cycle; } \text { stance }=\% \text { of gait cycle; } \\
\text { double stance }=\%\end{array}$ & $\begin{array}{l}\text { Isometric knee extension: } 56 \text { to } \\
54 \mathrm{ft} / \mathrm{lb} \text { ), balance measures (e.g., one- }\end{array}$ \\
\hline & & & $\begin{array}{l}\text { group (HOME) } \\
(n=39)\end{array}$ & & Coordination $/$ response $=\mathrm{msec}$ & measures ( $\left.p^{\prime} s>0.05\right)$; significant \\
\hline & & & Mena age, 83 & & & $\begin{array}{l}\text { (e.g., should flexion, } 159 \text { to } 161 \mathrm{~mm} \text { ), } \\
\text { balance, gait, coordination/response } \\
\text { time (351 to } 417 \text { msecs) ( } p^{\prime} \text { < }<0.05 \text { ) }\end{array}$ \\
\hline DeVito et al., 2000 [57] & Physical health & Pre-post test & $N=105$ & Duration, 8-10 months & Mobility measures score & Intervention group = significant \\
\hline & & & $\begin{array}{l}\text { Had a hospital } \\
\text { admission lasting } \\
2 \text { days or more or }\end{array}$ & $\begin{array}{l}\text { Intervention group }=24 \\
\text { sessions ( } 45 \text { min) of } 3 \text { sets of } \\
\text { low-intensity standard exercise }\end{array}$ & Gait score & $\begin{array}{l}\text { Balance score: } 9.6 \text { to 12.8) ( } p \text { 's < } \\
0.001 \text { ) }\end{array}$ \\
\hline & & & had been on bed & modalities (3 times a week) & Balance score & Control group = significant \\
\hline & & & $\begin{array}{l}\text { rest for } 2 \text { days or } \\
\text { more within the past } \\
1 \text { month }\end{array}$ & $\begin{array}{l}\text { targeting on flexibility, postural } \\
\text { stability, balance and gait (e.g., } \\
\text { extend leg up then back }\end{array}$ & Muscle strength score & $\begin{array}{l}\text { improvements in all outcomes (e.g., } \\
\text { Balance score: } 9.8 \text { to 10.4) (p's } \\
0.001 \text { ) }\end{array}$ \\
\hline & & & Male, 47; Female, 58 & $\begin{array}{l}\text { down, raise up and down on } \\
\text { toes then heels, march in place }\end{array}$ & & Between groups = intervention \\
\hline & & & $\begin{array}{l}\text { Intervention group } \\
(n=60)\end{array}$ & $\begin{array}{l}\text { etc.); continue performing } \\
\text { exercise until } 1 \text { year after the }\end{array}$ & & $\begin{array}{l}\text { group nas significant greater } \\
\text { samples in improving in gait, } \\
\text { balance and mobility measures ( } p \text { 's }<\end{array}$ \\
\hline & & & Mean age, 80 & Individualization of the program & & 0.001). e.g., $35.1 \%$ of intervention \\
\hline & & & Control group $(n=45)$ & $\begin{array}{l}\text { according to participant's ability } \\
\text { and progress }\end{array}$ & & while $15.9 \%$ of control groups \\
\hline & & & Mean age, 81 & $\begin{array}{l}\text { Control group = usual } \\
\text { activities }\end{array}$ & & $\begin{array}{l}\text { increased in walking ability }(p< \\
0.001)\end{array}$ \\
\hline & & & & $\begin{array}{l}\text { Overall intervention } \\
\text { compliance = } 91 \%\end{array}$ & & \\
\hline Schnelle et al., 2003 [66] & Physical health & $\mathrm{RCT}$ & $N=190$ & Duration, 8 months & Medical conditions (dermatological, & Between groups = intervention \\
\hline & & & $\begin{array}{l}\text { Living in nursing } \\
\text { home }\end{array}$ & $\begin{array}{l}\text { Intervention group = engaged } \\
\text { into the low-intensity functional }\end{array}$ & $\begin{array}{l}\text { genitourinary, gastrointestinal, } \\
\text { respiratory, endocrine, neurological } \\
\text { and cardiovascular systems, falls, and }\end{array}$ & $\begin{array}{l}\text { group nas significant smaller number } \\
\text { of falls than the control group ( } p^{\prime}< \\
0.05) \text {; no difference on other medical }\end{array}$ \\
\hline & & & Male, 28; Female, 162 & $\begin{array}{l}\text { Oriented exercise program: } \\
\text { Functional Incidental Training }\end{array}$ & pain, psychiatric and nutritional & conditions and cost of treatment \\
\hline & & & $\begin{array}{l}\text { Intervention group } \\
(n=92)\end{array}$ & $\begin{array}{l}\text { (FIT) ( } 5 \text { days a week; every } 2 \mathrm{~h} \\
\text { between } 0800 \text { to } 1600)\end{array}$ & & \\
\hline
\end{tabular}


Table 2 Description of studies included in the review (Continued)

\begin{tabular}{|c|c|c|c|c|c|c|}
\hline & & & Mean age, 87.3 & & Cost of treatment & \\
\hline & & & $\begin{array}{l}\text { Control group } \\
(n=98)\end{array}$ & & & \\
\hline & & & Mean age, 88.6 & $\begin{array}{l}\text { Control group = received } \\
\text { usual care from NH staff; no } \\
\text { change in their physical } \\
\text { activity or other measures. }\end{array}$ & & \\
\hline & & & & $\begin{array}{l}\text { Overall intervention } \\
\text { compliance }=91 \%\end{array}$ & & \\
\hline \multirow[t]{9}{*}{ Morgan et al., 2004 [63] } & \multirow[t]{9}{*}{ Physical health } & \multirow[t]{9}{*}{ RCT } & $N=229$ & Duration, 8 weeks & \multirow{2}{*}{$\begin{array}{l}\text { Gait and balance (Tinetti's gait and } \\
\text { balance assessment measures) }\end{array}$} & \multirow{2}{*}{$\begin{array}{l}\text { Exercise group }=28.6 \% \text { participants } \\
\text { fell; risk of falling decreased with low } \\
\text { baseline physical function }(p<0.001) \text {; } \\
\text { increased fall risk with high physical } \\
\text { function }(p<0.001)\end{array}$} \\
\hline & & & \multirow{3}{*}{$\begin{array}{l}\text { Had a hospital } \\
\text { admission or bed rest } \\
\text { for } 2 \text { days or more } \\
\text { within the previous } \\
\text { month }\end{array}$} & \multirow[t]{2}{*}{$\begin{array}{l}\text { Exercise group }=\text { perform } \\
\text { chair-sitting exercise and } \\
\text { standing balance exercises; } 3 \\
\text { times per week. }\end{array}$} & & \\
\hline & & & & & $\begin{array}{l}\text { Self-reported functional status (SF-36) } \\
=\text { range, } 0-100\end{array}$ & $\begin{array}{l}\text { Control group }=30.9 \% \text { participants } \\
\text { fell }\end{array}$ \\
\hline & & & & $\begin{array}{l}\text { Control group }=\text { continue their } \\
\text { usual activities. }\end{array}$ & $\begin{array}{l}\text { Number of fall for } 1 \text { year after the } \\
\text { assessment }\end{array}$ & $\begin{array}{l}\text { Overall, } 29.7 \% \text { of the participants } \\
\text { reported a fall during study period }\end{array}$ \\
\hline & & & Male, 67; Female, 162 & \multirow{5}{*}{$\begin{array}{l}\text { Overall intervention } \\
\text { compliance }=70 \%\end{array}$} & & \\
\hline & & & $\begin{array}{l}\text { Exercise group } \\
(n=119)\end{array}$ & & & \\
\hline & & & Mean age, 81.0 & & & \\
\hline & & & $\begin{array}{l}\text { Control group } \\
(n=110)\end{array}$ & & & \\
\hline & & & Mean age, 80.1 & & & \\
\hline
\end{tabular}


test involving domains of flexibility, balance, body-handling skill, reaction speed, and coordination before and after the program. Results revealed significant improvements in all physical domains by low-intensity exercise [54]. Moreover, increases in sense of enjoyment and self-rated improvement in physical performance were also reported by the participants after low-intensity exercise.

Similar benefits were also evidenced in Morgan et al.'s study [63], where the researchers employed a physical restoration intervention consisting mainly of a series of low-intensity standing and sitting exercises among a group of clinically defined at-risk older adults (i.e., those who had either a hospital admission or bed rest for 2 days or more within the previous month). In the 1-year fall-tracking period after the study, only $29 \%$ of the study participants reported a fall. This study also [63] found that the low-intensity exercise was more effective for the clinically defined at-risk older participants. Benefits among healthy older adults, however, were questionable; the results suggested low-intensity exercise led to an increased fall risk among healthy elders [63]. One possible explanation for the increased fall risk is that those with high physical functioning may have a higher threshold (i.e., a muscle strength level at which the exercise program starts to be effective); therefore, the high-functioning older adults did not benefit from the small increase in muscle strength from the exercise program [80]. Another possible explanation may be that fall risk increases simply by increasing amount of activity [80-82].

It is noteworthy that Tai Chi, a traditional Chinese martial art consisting of a series of slow but continuous body movements [83], is widely accepted to be a lowintensity fall prevention exercise for both high- and lowfunctioning older adults [59, 83-87]. In 1 study included in this review, the effectiveness of a 6-month Tai Chi program and a 6-month stretching program with identical weekly schedules (three times per week) was compared [59]. This study [59] showed that participants in the Tai Chi group reported fewer falls, lower proportions of falls, and fewer injurious falls than those in stretching group after the 6-month exercise intervention period. Moreover, in contrast to the stretching group, the Tai Chi group also showed improvements in all measures of functional balance, physical performance, and reduced fear of falling [59]. This study added new evidence to support reports that showed certain low-intensity exercises may be better than others at sufficient intensity for reducing fall incidence.

Apart from physical health benefits, the present review also found strong evidence to support the cognitive health benefits of low-intensity exercise. Two of the included studies investigated the effect of low-intensity exercise on depressive symptoms [64, 67]. Both studies showed a reduction in depression symptoms after the low-intensity exercise intervention (with 1 study using the Hamilton Rating Scale of Depression and the other study using the 30-item Geriatric Depression Scale) [67]. These findings suggested that exercise of low-intensity levels might be of adequate intensity to prevent depression-a valuable insight for preventing and treating cognitive health problems among older adults. In Hong Kong, the prevalence of depression among older adults was $11.0 \%$ for men and $14.5 \%$ for women [88]. Globally, it is estimated that major depressive disorder would become the second most prevalent disease among elderly people by 2020 [89]. Depression inflicts enormous suffering on individuals, often promoting social isolation, insomnia, and decreased concentration. Serious depression may even lead to recurrent thoughts of death and suicidal ideation. It also poses various challenges to families and to the community. Physical treatment (e.g., pharmacological and electroconvulsive therapy) and psychosocial treatment are long established and traditional treatments for clinical depression [90]. However, some of these treatments also bring unpleasant side effects that interfere with patients' quality of life and reduce compliance with prescribed drug therapies [91]. Exercise may help alleviate some of these unpleasant side effects.

Numerous studies have shown that exercise has been effective in reducing depression symptoms [92-96], with moderate intensity exercise being the most frequently prescribed exercise intensity in these studies. Blumenthal et al. [92], for example, conducted an exercise intervention with moderate intensity walking (intensity up to 70 to $85 \%$ of maximum heart rate reserve) for the treatment of major depression among older adults. The authors concluded that moderate intensity walking may be a viable treatment for major depression. Beyond moderate intensity exercise alone, however, low-intensity exercise and physical activity, in general, may also be effective means of coping with depression. In Singh et al.'s study [57], 8 weeks of high-intensity progressive resistance training ( $80 \%$ of one repetition maximum) was observed to be more effective than the low-intensity training ( $20 \%$ of one repetition maximum) for alleviating depression symptoms; however, lowintensity exercise was reported to significantly reduce the number of depressive symptoms by $29.0 \%$. Similar results were also displayed in Motl et al.'s study [64], where both low-intensity exercise groups and walking groups displayed a significant reduction in depressive symptoms after the 6-month intervention and in the 12-month and 60-month follow-up periods [64]. These findings indicated that moderate or vigorous intensity levels of physical activity might not be necessary to achieve cognitive health benefits. Implications for healthcare professionals may include increasing prescription of low-intensity exercise among older adults with cognitive health problems. More research should be 
conducted to confirm the relationship between lowintensity exercise and cognitive health.

This review also evaluated the quality of the selected studies with the established quality index [53] and included 10 RCTs. Among the studies included in this systematic review, the methodological quality of studies was generally modest (mean score $=18.69$, out of a highest possible score of 27). The quality index scale items with the highest scores in these studies were related to the reporting and external validity criteria. These items indicate strength in the clarity of the objective, main outcomes, participants' characteristics, main findings, and the representatives of the participants to the whole general population. Scale items that were less satisfied, however, were those related to internal validity-confounding-particularly pertaining to allocation concealment and inadequate adjustment for confounding in the analyses. Blinding of participants and researchers tends to be difficult owing to the nature of interventions.

\section{Limitation}

The findings of this review should be interpreted with consideration of some limitations. This review did not offer quantitative analyses (e.g., intention-to-treat and metaanalysis) on the effectiveness of low-intensity exercise due to the heterogeneity of the study designs. Five out of 15 studies were quasi-experimental studies in which participants were not randomly assigned to experimental or control groups; the efficacy of the quantitative analyses in yielding meaningful results may have thus been limited. Nevertheless, we included these studies in the review to enable a more comprehensive synthesis of the evidence. Although the quasi-experimental designs may have weakened the reliability of their study findings, it should be noted that the internal validity of the five quasi-experimental studies were scored as four $[61,64]$ to five $[56,57,60]$, at moderate levels of quality (Table 1). High-quality RCT designs are strongly suggested for future research on the topic of low-intensity exercise and older adults to overcome this limitation. Another limitation of this study was the unavailability of sample size calculation, which may also affect the validity and reliability of the study findings. Future research endeavors would do well to include sample sizes and power calculations. Finally, a considerable portion of the included studies did not assess the intervention compliance rate, one of the potential key differences between exercises of different intensities [97-99]. It is therefore also suggested that future research provide such information.

\section{Conclusions}

The findings from this review indicated that lowintensity exercise might offer both physical and cognitive health benefits to older adults aged 65 to 85 years-particularly among women, as shown in most of the included studies. Exercise could be of varying types, including but not limited to chair-sitting exercises, Tai Chi, walking, or stretching. In studies with high-risk populations (such as physically frail elders, nursing home residents, and fallers), low-intensity exercise intervention was useful in eliciting the desired physical and cognitive improvements. This finding is important, as most of the existing literature has focused on the benefits of moderate- and high-intensity exercise rather than those of low-intensity exercise. If low-intensity exercise is effective in promoting physical and cognitive health among older adults, it may be preferable when considering factors such as fall risk, safety, compliance, and effectiveness. Indeed, 7 of the included studies in the present review showed a satisfactory level of exercise intervention compliance rate for low-intensity exercise (>70 \%) $[53,59,64-68]$, whereas the remaining included studies did not report such information. It is, therefore, suggested that the exercise compliance of low-intensity exercise may be better than that of moderate- and high-intensity exercise, yielding better health benefits [97-99]. Further clinical application of lowintensity exercise still needs to be confirmed with additional research exploring best techniques and protocols for clinical populations. Incorporating cognitive training into low-intensity exercise programs (similar to Tai Chi) may also be a worthy research direction for future investigations.

\section{Appendix 1}

\section{Quality Index [53]}

Reporting: Were the following clearly described? $(\mathrm{Y} / \mathrm{N})$

1. Study hypothesis/aim/objective

2. Main outcomes

3. Characteristics of the participants

4. Interventions of interest

5. Distributions of principal confounders in each group

6. Main findings

7. Estimates of random variability for main outcomes

8. All the important adverse events that may be a consequence of intervention

9. Characteristics of patients lost to follow-up

10. Actual probability values for main outcomes

External validity (Y/N/unable to determine)

11. Were participants who were asked to participate representative of the entire population from which they were recruited?

12. Were participants who were prepared to participate representative of the entire population from which they were recruited?

13. Were the staff, places, and facilities representative of the treatment the majority of participants received? 
Internal validity - bias ( $\mathrm{Y} / \mathrm{N} /$ unable to determine)

14. Was an attempt made to blind participants to the intervention they received?

15. Was an attempt made to blind those measuring main outcomes of the intervention?

16. If any of the results of the study were based on "data dredging" was this made clear?

17. In trials and cohort studies, do analyses adjust for different lengths of follow-up? Or, in case-control studies, is the period between intervention and outcome the same for cases and controls?

18. Were appropriate statistical tests used to assess the main outcomes?

19. Was compliance with the intervention reliable?

20. Were main outcome measures reliable and valid? Internal validity - confounding (selection bias) $(\mathrm{Y} / \mathrm{N} /$ unable to determine)

21. For trials and cohort studies, were patients in different intervention groups? For case-control studies, were cases and controls recruited from the same population?

22. For trials and cohort studies, were participants in different intervention groups? For case-control studies, were cases and controls recruited over the same period of time?

23. Were participants randomized to intervention groups?

24. Was the randomized intervention assignment concealed from both patients and staff until recruitment was complete and irrevocable?

25. Was there adequate adjustment for confounding in the analyses from which main findings were drawn?

Power

27. Did the study have sufficient power to detect a clinically important effect where the probability for a difference due to chance was less than $5 \%$ ?

\section{Competing Interests}

The authors declare that they have no competing interests.

\section{Authors' Contributions}

The first author ACYT carried out the screenings, reviews and analyses of the articles, drafted and revised the manuscript. The second and third authors TWLW and PHL have carried out screenings and reviews of the articles. All authors read and approved the final manuscript.

\section{Acknowledgement}

We are appreciative of the support given by Mr. Sadahiro Omuro, MA, for resolving the rating discrepancies in the quality index assessment.

\section{Author details}

'Department of Health and Physical Education, Hong Kong Institute of Education, Tai Po, Hong Kong, China. ${ }^{2}$ Institute of Human Performance, The University of Hong Kong, Pokfulam, Hong Kong, China. ${ }^{3}$ School of Nursing, Hong Kong Polytechnic University, Hunghum, Hong Kong, China.

Received: 4 September 2015 Accepted: 15 September 2015

Published online: 20 October 2015

\section{References}

1. Anderson GF, Hussey PS. Population aging: a comparison among industrialized countries. Health Affairs. 2000;19:191-203.

2. Badley EM, Wang PP. Arthritis and the aging population: projections of arthritis prevalence in Canada 1991 to 2031. J Rheumatol. 1998;25:138-44.

3. Meng X, D'Arcy C. Successful aging in Canada: prevalence and predictors from a population-based sample of older adults. Gerontology. 2013;60:65-72.

4. Muniz-Terrera G, van den Hout RA, Piccinin AM, Matthews FE, Hofer SM. Investigating terminal decline: results from a UK population-based study of aging. Psychol Aging. 2013;28:377.

5. Sheets DJ, Gallagher EM. Aging in Canada: state of the art and science. The Gerontologist. 2013;53:1-8.

6. Stewart S, Maclntyre K, Capewell S, McMurray JJV. Heart failure and the aging population: an increasing burden in the 21st century? Heart. 2003:89:49-53.

7. Wang Y, Graham C, Colditz A, Kuntz KM. Forecasting the obesity epidemic in the aging US population. Obesity. 2007;15:2855-65.

8. Census and Statistics Department, HKSAR Hong Kong Population Project 2012-2041. Census and Statistics Department, 2012. http:// www.statistics.gov.hk/pub/B71208FB2012XXXXB0100.pdf. Accessed 11 Aug 2014.

9. Tanaka H, Seals DR. Age and gender interactions in physiological functional capacity: insight from swimming performance. J Appl Physio. 1997:82:846-51.

10. Buchner DM, Wagner EH. Preventing frail health. Clin Geriatr Med. 1992;8:1-17.

11. Jette $A M$, Branch LG, Berlin J. Musculoskeletal impairments and physical disablement among the aged. J Gerontol. 1990;45:203-8.

12. Census and Statistics Department. Socio-demographic profile, health status and self-care capability of older persons, thematic household survey report No. 40. Hong Kong: HKSAR 2009.

13. Khaw KT. How many, how old, how soon? Bri Med J. 1999;319:1350.

14. Rubenstein LZ. Falls in older people: epidemiology, risk factors and strategies for prevention. Age Ageing. 2006;35:37-41.

15. Chiu HFK, Lam LCW, Chi I, Leung T, Li SW, Law WT, et al. Prevalence of dementia in Chinese elderly in Hong Kong. Neurology. 1998:50:1002-9.

16. Chu LW. Alzheimer's disease: early diagnosis and treatment. Hong Kong Med J. 2012;18:228-37.

17. Fisher GG, Stachowski A, Infurna FJ, Faul JD, Grosch J, Tetrick LE. Mental work demands, retirement, and longitudinal trajectories of cognitive functioning. J Occup Health Psychol. 2014;19:231.

18. Plassman $B L$, Langa KM, Fisher GG, Heeringa $S G$, Weir DR, Ofstedal MB, et al. Prevalence of cognitive impairment without dementia in the United States. Ann Intern Med. 2008;148:427-34.

19. Wortmann M. Dementia: a global health priority-highlights from an ADI and World Health Organization report. Alzheimers Res Ther. 2012:4:40.

20. Der G, Batty GD, Deary IJ. The association between IQ in adolescence and a range of health outcomes at 40 in the 1979 US national longitudinal study of youth. Intelligence. 2009:376:573-80.

21. González HM, Bowen ME, Fisher GG. Memory decline and depressive symptoms in a nationally representative sample of older adults: the health and retirement study (1998-2004). Dement Geriatr Cogn Disord. 2008;25:266-71.

22. HKSAR. The 2013-2014 Budget. HKSAR [Internet] 2013. [Cited 2014 Aug 11]. Available from http://www.budget.gov.hk/2013/eng/budget27.html.

23. Chou $\mathrm{CH}$, Hwang $\mathrm{CL}$, Wu YT. Effect of exercise on physical function, daily living activities, and quality of life in the frail older adults: a meta-analysis. Arch Phys Med Rehabil. 2012;93:237-44.

24. Manson JE, Hu FB, Rich-Edwards JW, Colditz GA, Stampfer MJ, Willett WC, et al. A prospective study of walking as compared with vigorous exercise in the prevention of coronary heart disease in women. New Engl J Med. 1999;341:650-58.

25. Mazzeo R, Tanaka H. Exercise prescription for the elderly. Sports Med. 2001;31:809-18.

26. Pedersen BK, Saltin B. Evidence for prescribing exercise as therapy in chronic disease. Scand J Med Sci Spor. 2006;16:3-63.

27. Roberts CK, Barnard RJ. Effects of exercise and diet on chronic disease. J Appl Physio. 2005;98:3-30.

28. Taylor AH, Cable NT, Faulkner G, Hillsdon M, Narici M, Van Der Bij AK Physical activity and older adults: a review of health benefits and the effectiveness of interventions. J Sports Sci. 2004;22:703-25.

29. Caspersen CJ, Powell KE, Christenson GM. Physical activity, exercise, and physical fitness: definitions and distinctions for health-related research. Public health Rep. 1985;2:126. 
30. Nieman DC. Exercise testing and prescription. A health-related approach 5th ed. Mountain View (CA): Mayfield 2003: 63-5

31. Barnett A, Smith B, Lord SR, Williams M, Baumand A. Community-based group exercise improves balance and reduces falls in at-risk older people: a randomised controlled trial. Age Ageing. 2003:32:407-14.

32. Reinsch $S$, MacRae P, Lachenbruch PA, Tobis JS. Attempts to prevent falls and injury: a prospective community study. The Gerontologist. 1992;32:450-56.

33. Sturnieks DL, St George R, Lord RS. Balance disorders in the elderly. Neurophysiol Clin. 2008:38:467-78.

34. Weerdesteyn V, Rijken H, Geurts ACH, Smits-Engelsman BCM, Mulder T, Duysens J. A five-week exercise program can reduce falls and improve obstacle avoidance in the elderly. Gerontology. 2006;52:131-41.

35. Heyn P, Abreu BC, Ottenbacher KJ. The effects of exercise training on elderly persons with cognitive impairment and dementia: a meta-analysis. Arch Phys Med Rehabil. 2004;85:1694-704.

36. Ruuskanen JM, Ruoppila I. Physical activity and psychological well-being among people aged 65 to 84 years. Age Ageing. 1995;24:292-96.

37. Uffelen JGZ, Paw MJM CA, Hopman-Rock M, Mechelen W. The effects of exercise on cognition in older adults with and without cognitive decline: a systematic review. Clin J Sport Med. 2008;18:486-500.

38. Armstrong L. ACSM's guidelines for exercise testing and prescription. Philadelphia: Lippincott Williams \& Wilkins; 2006.

39. Jette M, Sidney $K$, Blümchen G. Metabolic equivalents (METS) in exercise testing, exercise prescription, and evaluation of functional capacity. Clin Cardiol. 1990;13:555-65.

40. Ainsworth BE, Haskell WL, Herrmann SD, Meckes N, Bassett Jr DR, Tudor-Locke C, et al. 2011 Compendium of Physical Activities: a second update of codes and MET values. Med Sci Sports Exerc. 2011;43:1575-81.

41. Pollock ML, Gaesser GA, Butcher JD, Després JP, Dishman RK, Franklin BA, et al. ACSM position stand: the recommended quantity and quality of exercise for developing and maintaining cardiorespiratory and muscular fitness, and flexibility in healthy adults. Med Sci Sports Exerc. 1998:30:975-91.

42. Department of Health, HKSAR. Classification of physical activity and level of intensity. HKSAR,2011.http://www.change4health.gov.hk/en/ physical_avtivity/facts/classification/index.html. Accesed 11 Aug 2014.

43. World Health Organization. Global health risks: mortality and burden of disease attributable to selected major risks. World Health Organization 2009.

44. Physical Activity Guidelines Advisory Committee. Physical activity guidelines advisory committee report, 2008. Washington: US Department of Health and Human Services; 2008. p. 1-14.

45. Larson EB, Wang L, Bowen JD, McCormick WC, Teri L, Crane P, et al. Exercise is associated with reduced risk for incident dementia among Persons 65 years of age and older. Ann Intern Med. 2006;144:73-81.

46. Mather AS, Rodriguez C, Guthrie MF, Mcharg AM, Reid IC, McMurdo MET. Effects of exercise on depressive symptoms in older adults with poorly responsive depressive disorder: randomised controlled trial. Br J Psychiatry. 2002;180:411-15.

47. Sims J, Hill K, Davidson S, Gunn J, Huang N. Exploring the feasibility of a community-based strength training program for older people with depressive symptoms and its impact on depressive symptoms. BMC Geriatr. 2006:6:18.

48. Singh NA, Clements KM, Singh MAF. The efficacy of exercise as a long-term antidepressant in elderly subjects: a randomized controlled trial. J Gerontol Med Sci. 2001;56:497-504.

49. Seynnes $O$, Fiatarone Singh MA, Hue O, Pras $P$, Legros $P$, Bernard PL. Physiological and functional responses to low-moderate versus highintensity progressive resistance training in frail elders. J Gerontol Series A Biol Sci Med Sci. 2004;59:503-09.

50. Butcher LR, Backx ATK, Webb ALEDRR, Morris K. Low-intensity exercise exerts beneficial effects on plasma lipids via PPARF. Med Sci Sports Exerc. 2008;40:1263-70

51. Lee I, Rexrode KM, Cook NR, Manson JE, Buring JE. Physical activity and coronary heart disease in women: is "no pain, no gain" passé? JAMA. 2001;285:1447-54.

52. Moher D, Liberati A, Tetzlaff J, Altman DG. Preferred reporting items for systematic reviews and meta-analyses: the PRISMA statement. Ann Intern Med. 2009;159:264-9.

53. Downs SH, Black N. The feasibility of creating a checklist for the assessment of the methodological quality both of randomised and non-randomised studies of health care interventions. J Epidemiol Commun H. 1998;52:377-84.

54. Blair CK, Morey MC, Desmond RA, Cohen HJ, Sloane R, Snyder DC, et al. Light-intensity activity attenuates functional decline in older cancer survivors. Med Sci Sports Exerc. 2014;46:1375-83.
55. Brown M, Sinacore DR, Ehsani AA, Binder EF, Holloszy JO, Kohrt WM. LowIntensity exercise as a modifier of physical frailty in older adults. Arch Phys Med Rehabil. 2000:81:960-65.

56. Dawe D, Moore-Orr R. Low-intensity, range-of-motion exercise: invaluable nursing care for elderly patients. J Adv Nur. 1995;21:675-81.

57. DeVito CA, Morgan RO, Duque M, Abdel-Moty E, Virnig BA. Physical performance effects of low-intensity exercise among clinically defined highrisk elders. Gerontology. 2003;49:146-54.

58. Lam LCW, Chau RCM, Wong BML, Fung AWT, Lui WC, Tam CCW, et al. Interim follow-up of a randomized controlled trial comparing Chinese style mind body (Tai Chi) and stretching exercises on cognitive function in subjects at risk of progressive cognitive decline. Int J Geriatr. 2011;26:733-40.

59. Li F, Harmer P, Fisher KJ, McAuley E, Chaumeton N, Eckstrom E, et al. Tai Chi and fall reductions in older adults: a randomized controlled trial. J Gerontol A Biol Sci Med Sci. 2005:60:187-94.

60. Mangione KK, McCully K, Gloviak A, Lefebvre I, Hofmann M, Craik R. The effects of high-intensity and low-intensity cycle ergometry in older adults with knee osteoarthritis. J Gerontol A Biol Sci Med Sci. 1999;54:184-90.

61. Means KM, Rodell DE, O'Sullivan PS, Cranford LA. Rehabilitation of elderly fallers: pilot study of a low to moderate intensity exercise program. Arch Phys Med Rehabil. 1996;77:1030-36.

62. Morey MC, Snyder DC, Sloane R, Cohen HJ, Peterson B, Hartman TJ, et al. Effects of home-based diet and exercise on functional outcomes among older, overweight long-term cancer survivors: renew: a randomized controlled trial. JAMA. 2009;301:1883-91.

63. Morgan RO, Virnig BA, Duque M, Abdel-Moty E, DeVito CA. Low-intensity exercise and reduction of the risk for falls among at-risk elders. J Gerontol A Biol Sci Med Sci. 2004;59:1062-67.

64. Motl R, Konopack J, McAuley E, Elavsky S, Jerome G, Marquez D. Depressive symptoms among older adults: Long-term reduction after a physical activity intervention. J Behav Med. 2005;28:385-94

65. Rosie J, Taylor D. Sit-to-stand as home exercise for mobility-limited adults over 80 years of age-GrandStand SystemTM may keep you standing? Age and Ageing. 2007;36:555-62.

66. Schnelle JF, Kapur K, Alessi C, Osterweil D, Beck JG, Al-Samarrai NR, et al. Does an exercise and incontinence intervention save healthcare costs in a nursing home population? J Am Geriatr Soc. 2003;51:161-68.

67. Singh NA, Stavrinos TM, Scarbek Y, Galambos G, Liber C, Fiatarone Singh MA. A randomized controlled trial of high versus low intensity weight training versus general practitioner care for clinical depression in older adults. J Gerontol A Biol Sci Med Sci. 2005;60:768-76.

68. Wolfson L, Whipple R, Derby C, Judge J, King M, Amerman P, et al. Balance and strength training in older adults: intervention gains and Tai Chi maintenance. J Am Geriatr Soc. 1996;44:498-506.

69. Hausdorff JM, Rios DA, Edelberg HK. Gait variability and fall risk in community-living older adults: a 1-year prospective study. Arch Phys Med Rehabil. 2001:82:1050-56.

70. Kannus P, Parkkari J, Koskinen S, Niemi S, Palvanen M, Järvinen M, et al. Fallinduced injuries and deaths among older adults. JAMA. 1999;281:1895-99.

71. Sattin RW. Falls among older persons: a public health perspective. Annu Rev Public Health. 1992;13:489-508.

72. Chu LW, Chiu AYY, Chi I. Falls and subsequent health service utilization in community-dwelling Chinese older adults. Arch Gerontol Geriatr. 2008;46:125-35.

73. Nevitt MC, Cummings SR, Kidd S, Black D. Risk factors for recurrent nonsyncopal falls: a prospective study. JAMA. 1989;261:2663-68.

74. Tinetti ME, Speechley M, Ginter SF. Risk factors for falls among elderly persons living in the community. New Engl J Med. 1988;319:1701-7.

75. Close J, Ellis M, Hooper R, Glucksman E, Jackson S, Swift C. Prevention of falls in the elderly trial (PROFET): a randomised controlled trial. The Lancet. 1999;353:93-7.

76. Tinetti ME, Doucette J, Claus E, Marottoli R. Risk factors for serious injury during falls by older persons in the community. J Am Geriatr Soc. 1995:43:1214-1221.

77. Biderman A, Cwikel J, Fried AV, Galinsky D. Depression and falls among community dwelling elderly people: a search for common risk factors. J Epidemiol Com H. 2002;56:631-6.

78. Tinetti ME, Richman D, Powell L. Falls efficacy as a measure of fear of falling. J Gerontol. 1990;45:239-43.

79. Vellas BJ, Wayne SJ, Romero LJ, Baumgartner RN, Garry PJ. Fear of falling and restriction of mobility in elderly fallers. Age Ageing. 1997;26:189-93. 
80. Gardner MM, Robertson MC, Campbell AJ. Exercise in preventing falls and fall related injuries in older people: a review of randomised controlled trials. Brit J Sport Med. 2000;34:7-17.

81. King MB, Tinetti ME. Falls in community-dwelling older persons. J Am Geriatr Soc. 1995:43:1146-54.

82. Rubenstein LZ, Josephson KR, Trueblood PR, Loy S, Harker JO, Pietruszka FM, et al. Effects of a group exercise program on strength, mobility, and falls among fall-prone elderly men. J Gerontol A Biol Sci Med Sci. 2000;55:317-21.

83. Wu G. Evaluation of the effectiveness of Tai Chi for improving balance and preventing falls in the older population-a review. J Am Geriatr Soc. 2002;50:746-54.

84. Choi JH, Moon J-S, Song R. Effects of Sun-style Tai Chi exercise on physical fitness and fall prevention in fall-prone older adults. J Adv Nur. 2005;51:150-7.

85. Logghe IHJ, Verhagen AP, Rademaker ACHJ, Bierma-Zeinstra SMA van Rossum E, Faber MJ, et al. The effects of Tai Chi on fall prevention, fear of falling and balance in older people: a meta-analysis. Prev Med. 2010;51:222-7.

86. Province MA, Hadley EC, Hornbrook MC, Lipsitz LA, Miller JP, Mulrow CD, et al. The effects of exercise on falls in elderly patients: a preplanned meta-analysis of the ficsit trials. JAMA. 1995;273:1341-7.

87. Wolf SL, Coogler C, Xu T. Exploring the basis for Tai Chi Chuan as a therapeutic exercise approach. Arch Physl Med Rehabil. 1997;78:886-92.

88. Chi I, Yip PSF, Chiu HFK, Chou KL, Chan KS, Kwan CW, et al. Prevalence of depression and its correlates in Hong Kong's Chinese older adults. Am J Geriatr Psychiatry. 2005;13:409-16.

89. Chapman DP, Perry GS. Peer reviewed: depression as a major component of public health for older adults. Prev Chronic Dis. 2008:5:1-9.

90. Beck AT, Alford BA. Depression: causes and treatment. Philadelphia: University of Pennsylvania Press; 2009

91. Barbour KA, Blumenthal JA. Exercise training and depression in older adults. Neurobiol Aging. 2005;26:119-23.

92. Blumenthal JA, Babyak MA, Moore KA, Craighead WE, Herman S, Khatri P, et al. Effects of exercise training on older patients with major depression. Arch Intern Med. 1999;159:2349-56.

93. Ensari I, Motl RW, Pilutti LA. Exercise training improves depressive symptoms in people with multiple sclerosis: results of a meta-analysis. J Psychosom Res. 2014;76:465-71.

94. Dunn AL, Trivedi MH, Kampert JB, Clark CG, Chambliss HO. Exercise treatment for depression: efficacy and dose response. Am J Prev Med. 2005;28:1-8.

95. Josefsson T, Lindwall M, Archer T. Physical exercise intervention in depressive disorders: meta-analysis and systematic review. Scand J Med Sc Spor. 2014;24:259-72.

96. McNeil JK, LeBlanc EM, Joyner M. The effect of exercise on depressive symptoms in the moderately depressed elderly. Psychol Aging. 1991;6:487.

97. Pollock ML, Franklin BA, Balady GJ, Chaitman BL, Fleg JL, Fletcher B, et al. Resistance exercise in individuals with and without cardiovascular disease: benefits, rationale, safety, and prescription an advisory from the committee on exercise, rehabilitation, and prevention, council on clinical cardiology, American Heart Association. Circulation. 2000;101:828-33.

98. Allen K, Morey M. Physical activity and adherence. In: Bosworth H, editor. Improving Patient Treatment Adherence. New York: Springer; 2010. p. 9-38.

99. King AC, Rejeski WJ, Buchner DM. Physical activity interventions targeting older adults: a critical review and recommendations. American Journal of Preventive Medicine. 1998;15:316-33.

\section{Submit your manuscript to a SpringerOpen ${ }^{\circ}$ journal and benefit from:}

- Convenient online submission

- Rigorous peer review

- Immediate publication on acceptance

- Open access: articles freely available online

- High visibility within the field

- Retaining the copyright to your article

Submit your next manuscript at $>$ springeropen.com 